\title{
Electroweak corrections
}

\section{to gauge-boson pair production processes at the LHC including leptonic W/Z decays}

\author{
Benedikt Biedermann \\ Julius-Maximilians-Universität Würzburg, Institut für Theoretische Physik und Astrophysik \\ E-mail: benedikt.biedermann@physik.uni-wuerzburg.de
}

\section{Marina Billoni}

Eberhard Karls Universität Tübingen, Institut für Theoretische Physik

E-mail: marina.billoni@gmail.com

\section{Ansgar Denner}

Julius-Maximilians-Universität Würzburg, Institut für Theoretische Physik und Astrophysik

E-mail: ansgar.denner@physik. uni-wuerzburg.de

\section{Stefan Dittmaier}

Universität Freiburg, Physikalisches Institut

E-mail: stefan.dittmaierephysik.uni-freiburg.de

\section{Lars Hofer}

Universitat de Barcelona (UB), Department de Física Quàntica i Astrofísica (FQA)

E-mail: lhofer@ifae.es

\section{Barbara Jäger}

Eberhard Karls Universität Tübingen, Institut für Theoretische Physik

E-mail: barbara.jaegereitp.uni-tuebingen. de

\section{Lukas Salfelder}

Eberhard Karls Universität Tübingen, Institut für Theoretische Physik

E-mail: lukas.salfelder@uni-tuebingen.de

We briefly report on results of the first calculations of next-to-leading-order electroweak corrections to $\mathrm{W}$ - and Z-boson pair production at the LHC that fully take into account leptonic $\mathrm{W} / \mathrm{Z}$ boson decays and off-shell effects. For W-pair production we generally confirm the validity of a double-pole approximation based on doubly-resonant contributions, but also reveal its failure to describe transverse-lepton-momentum distributions in the high-energy tails. For Z-pair production we zoom into the $\mathrm{ZZ}^{*}$ off-shell region, where direct $\mathrm{Z}$-pair production delivers irreducible background to the Higgs-boson signal, and find that electroweak corrections cannot be trivially extrapolated from the on-shell $\mathrm{ZZ}$ region.

Loops and Legs in Quantum Field Theory

24-29 April 2016

Leipzig, Germany

\footnotetext{
*Speaker.
} 


\section{Introduction}

Precision studies of pair production processes of the massive electroweak (EW) gauge bosons $\mathrm{W}$ and $\mathrm{Z}$ are and will continue to be phenomenologically very interesting at the Large Hadron Collider (LHC). They do not only offer an indirect window to potential new-physics effects through their sensitivity to the triple-gauge-boson couplings, but also represent sources of irreducible background to many new-physics searches and to precision studies of the Higgs boson. Owing to their clean experimental signature, leptonically decaying $\mathrm{W} / \mathrm{Z}$ bosons are of primary interest in this context. In order to match and possibly exceed the accuracy in the experimental analyses, the precision of theoretical predictions has to reach the level of few percent, a task that requires the inclusion of higher-order corrections of the strong and EW interactions and of decay and off-shell effects of the $\mathrm{W} / \mathrm{Z}$ bosons.

In recent years, precision QCD calculations for W/Z-boson pair production with leptonic decays have been pushed to the next-to-next-to-leading order (NNLO) level [1] and beyond fixed perturbative orders by QCD resummations [2], parton-shower matching [3], and multi-jet merging [4]. Starting at NNLO, $\mathrm{W}$ and $\mathrm{Z}$ pairs can also be produced in gluon-gluon scattering via quark loops [5], a channel that is particularly important as background to Higgs production. Very recently, next-to-leading order (NLO) predictions for $\mathrm{gg} \rightarrow \mathrm{WW} / \mathrm{ZZ}$ were presented in Ref. [6]. Electroweak corrections at NLO were first calculated for stable $\mathrm{W} / \mathrm{Z}$ bosons $[7,8]$ and subsequently extended to leptonically decaying $\mathrm{W}$ bosons including off-shell effects in the resonance region in the so-called double-pole approximation (DPA) [9]. ${ }^{1}$ Most recently, the calculations of EW corrections were generalized to fully include leptonic $\mathrm{W} / \mathrm{Z}$ decay and off-shell effects upon employing complete $2 \rightarrow 4$ particle NLO amplitudes [11, 12].

In the following we briefly review the salient features and central results of our NLO EW calculations for the processes $\mathrm{pp} \rightarrow v_{\mu} \mu^{+} \mathrm{e}^{-} \bar{v}_{\mathrm{e}}+X$ [12] and $\mathrm{pp} \rightarrow \mu^{+} \mu^{-} \mathrm{e}^{+} \mathrm{e}^{-}+X$ [11]. Specifically, we compare results from the full four-fermion $(4 f)$ calculation to previous results on W-pair production obtained in DPA [9], putting particular emphasis on high momentum transfer where EW corrections are generically very pronounced. Moreover, we inspect the EW corrections to ZZ production in closer detail where direct $\mathrm{Z}$-pair production appears as background to the $\mathrm{H} \rightarrow 4$ leptons decay of the Higgs boson, i.e. the process of $\mathrm{ZZ}^{*}$ production where at most one $\mathrm{Z}$ boson can be on shell.

\section{Calculating NLO corrections to $\mathrm{pp} \rightarrow \mathrm{WW} / \mathrm{ZZ} \rightarrow 4$ leptons}

We consider four-lepton production in proton-proton collisions which at leading order (LO) involves the partonic processes

$$
\bar{q} q / q \bar{q} / \gamma \gamma \rightarrow \ell_{1} \bar{\ell}_{2} \ell_{3} \bar{\ell}_{4} .
$$

Specifically, we take $v_{\mu} \mu^{+} \mathrm{e}^{-} \bar{v}_{\mathrm{e}}$ and $\mu^{+} \mu^{-} \mathrm{e}^{+} \mathrm{e}^{-}$final states as representatives for $\mathrm{W}$ - and Z-pair production, respectively. The $\bar{q} q / q \bar{q}$ channels (simply called $\bar{q} q$ in the following) involve all five

\footnotetext{
${ }^{1}$ Similarly, EW corrections to leptonically decaying W/Z pairs are included in HERWIG [10], though, in an approach that approximately integrates out photons emitted in the pair production process, i.e. their kinematics is not transferred to the events.
} 


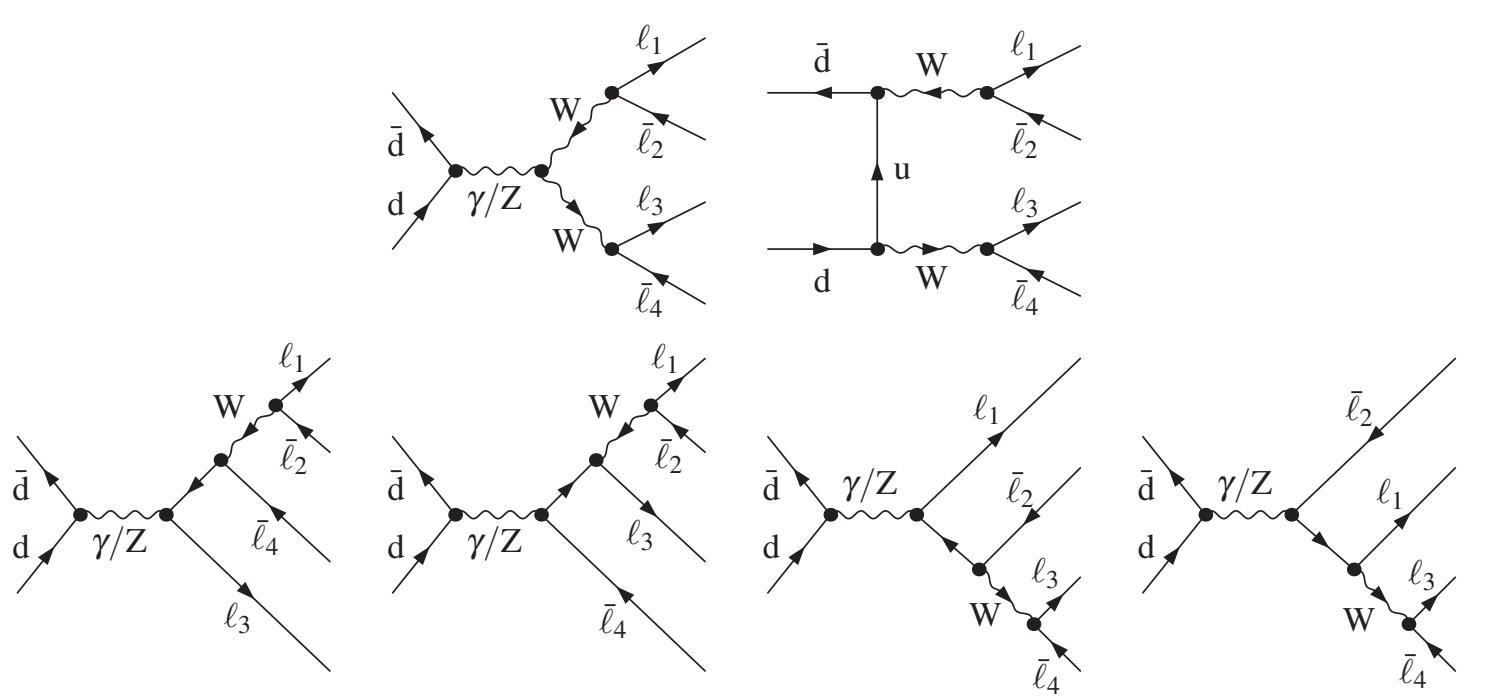

Figure 1: Tree-level diagrams for the partonic (charged-current) process $\overline{\mathrm{d}} \mathrm{d} \rightarrow 4$ leptons.
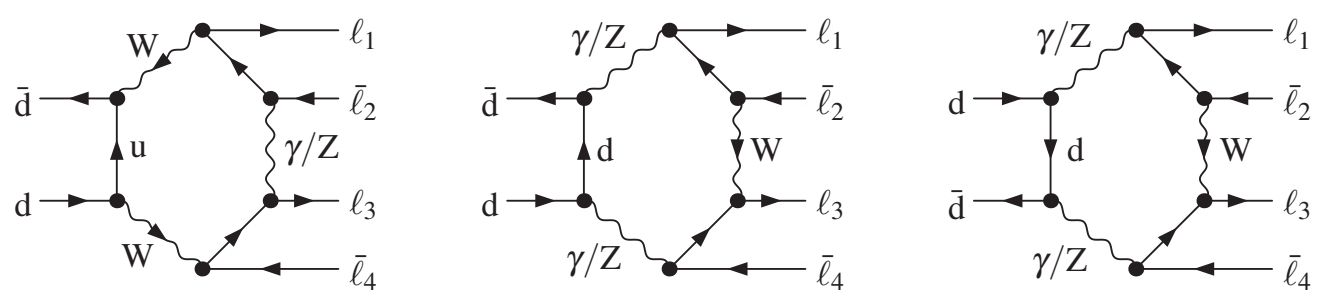

Figure 2: Some hexagon diagrams for the partonic (charged-current) process $\overline{\mathrm{d}} \mathrm{d} \rightarrow 4$ leptons.

light quarks species in the initial state and receive doubly-resonant contributions from WW or ZZ production for partonic centre-of-mass energies $\sqrt{\hat{s}}>2 M_{V}(V=\mathrm{W}, \mathrm{Z})$, respectively. Exemplarily, we show the complete set of tree-level diagrams for one partonic $\bar{q} q$ channel in Fig. 1, illustrating the fact that there are diagrams with 2 , 1 , or 0 potentially resonant propagators. ${ }^{2}$ The $\gamma \gamma$ channels nominally contribute at $\mathrm{LO}$ as well, but are suppressed w.r.t. to $\bar{q} q$ contributions because of the smallness of the photon distribution function of the proton. We, thus, include this contribution as correction in our predictions.

NLO EW corrections comprise purely virtual one-loop diagrams and real corrections with one additional external photon. Figure 2 illustrates the most complicated one-loop topology (so-called hexagon diagrams) for a specific partonic channel. The real photonic corrections are classified into bremsstrahlung corrections with $4 \ell+\gamma$ final states, and photon-induced contributions with $q \gamma / \gamma q / \bar{q} \gamma / \gamma \bar{q}$ initial states (simply called $q \gamma$ in the following) and an additional $q / \bar{q}$ in the final state. We base our predictions on all $\bar{q} q$ contributions at NLO, supplemented by the tree-level $\gamma \gamma$ and $q \gamma$ contributions for $\mathrm{W}$-pair production. For ZZ production, channels with photons in the initial state turn out to be phenomenologically negligible.

We have performed two independent calculations of all contributions which are numerically cross-checked both at the level of amplitudes and cross sections. One calculation closely fol-

\footnotetext{
${ }^{2}$ Non-resonant contributions exist for neutral-current channels.
} 
lows the diagrammatic approach described in Ref. [13] where NLO EW corrections to $\mathrm{e}^{+} \mathrm{e}^{-} \rightarrow$ 4 fermions via W-boson pairs were calculated. The other calculation has been carried out with the program RECOLA [14] allowing for the automated generation of NLO EW amplitudes. Both loop calculations employ the library COLLIER [15] which is based on the results of Ref. [16] to evaluate all one-loop integrals with complex W/Z masses with sufficient numerical stability.

Infrared (soft and/or collinear) singularities are treated in the dipole subtraction approach for photon radiation [17]. Muons are considered to be fully isolated ("bare") from collinear photons, but electrons are recombined with photons in some collinear radiation cone ("dressed leptons"). Some of the presented observables are, thus, collinear unsafe w.r.t. photon radiation off muons and involve mass-singular photonic corrections $\propto(\alpha / \pi) \ln \left(m_{\mu} / Q\right)$, where $Q$ is some hard scale. All observables, however, are collinear safe w.r.t. photon radiation off electrons, where the collinearly enhanced photonic corrections are logarithmically sensitive to the resolution parameter of the recombination cone.

In order to achieve a gauge-invariant description of the $\mathrm{W} / \mathrm{Z}$ resonances that supports NLO accuracy everywhere in phase space, we employ the complex-mass scheme [13], which replaces the real W- and Z-boson masses by complex quantities, including also the corresponding complexification of EW couplings.

\section{Results on $\mathbf{W}$-pair production: full $2 \rightarrow 4$ matrix elements versus double-pole approximation}

In Ref. [12] we have discussed the EW corrections to $\mathrm{pp} \rightarrow v_{\mu} \mu^{+} \mathrm{e}^{-} \bar{v}_{\mathrm{e}}+X$ in detail, considering three different event-selection procedures: an "inclusive" setup with identification cuts only, a second setup specifically tuned to W-pair analyses ("ATLAS WW"), and a setup that is optimized for Higgs-boson analyses. The NLO EW corrections to integrated cross sections are at the level of few percent, with photon-induced contributions at or below the percent level. While the EW corrections to angular and rapidity distributions still stay at the level of some percent, the corrections to invariant-mass and transverse-momentum distributions generically grow to some $-10 \%$ in the $\mathrm{TeV}$ range, as expected by the dominance of the universal EW high-energy logarithms. In the TeV range photon-induced corrections contribute some percent to the cross sections.

In Ref. [12] we have, in particular, analyzed the validity and quality of the DPA for W-pair production, which was constructed for the virtual EW corrections in Ref. [9]. The DPA described there employs full matrix elements for all LO contributions and real-photonic corrections and applies the pole expansion only to the virtual corrections, following the approach implemented in the Monte Carlo generator RACOONWW [18] for $\mathrm{e}^{+} \mathrm{e}^{-} \rightarrow \mathrm{WW} \rightarrow 4 f$ at LEP2 and the ILC. The relative theoretical uncertainty $\Delta_{\mathrm{DPA}}$ of the DPA is not only given by the typical size of missing higher-order corrections, but also set by the intrinsic uncertainty of the pole expansion. Assuming that all LO contributions are based on full matrix elements and that the relative correction in DPA, $\delta_{\mathrm{EW}}^{\mathrm{DPA}}$, is normalized to the full $\mathrm{LO}$ cross section $\sigma_{\mathrm{LO}}$, i.e.

$$
\sigma_{\mathrm{NLOEW}}^{\mathrm{DPA}}=\sigma_{\mathrm{LO}}+\Delta \sigma_{\mathrm{EW}}^{\mathrm{DPA}}=\sigma_{\mathrm{LO}}\left(1+\delta_{\mathrm{EW}}^{\mathrm{DPA}}\right), \quad \delta_{\mathrm{EW}}^{\mathrm{DPA}}=\frac{\Delta \sigma_{\mathrm{EW}}^{\mathrm{DPA}}}{\sigma_{\mathrm{LO}}}
$$


we estimate $\Delta_{\mathrm{DPA}}$ to

$$
\Delta_{\mathrm{DPA}} \sim \max \{\left(\delta_{\mathrm{EW}}^{\mathrm{DPA}}\right)^{2}, \underbrace{\frac{\alpha}{\pi} \frac{\Gamma_{\mathrm{W}}}{M_{\mathrm{W}}} \ln (\ldots)}_{\lesssim 0.5 \%},\left|\delta_{\mathrm{EW}}^{\mathrm{DPA}}\right| \times \frac{\left|\sigma_{\mathrm{LO}}-\sigma_{\mathrm{LO}}^{\mathrm{DPA}}\right|}{\sigma_{\mathrm{LO}}^{\mathrm{DPA}}}\} .
$$

The first term on the r.h.s. of Eq. (3.2) corresponds to the missing higher-order EW corrections, similar to the NLO limitation of the full $4 f$ calculation, whose EW uncertainty can be estimated to $\Delta_{4 f} \sim \delta_{\mathrm{EW}}^{2}$, where $\delta_{\mathrm{EW}}$ is the relative NLO EW correction factor. The second term on the r.h.s. of Eq. (3.2) indicates the size of the off-shell contributions to the EW corrections in regions where the DPA applies. This estimate is based on the typical size of the respective effects: the off-shell contributions amounting to a fraction $\sim \Gamma_{\mathrm{W}} / M_{\mathrm{W}}$, and the EW corrections being of order $\sim \alpha / \pi$ times some moderate logarithmic factor (see also Refs. $[9,13,18]$ ). The last term on the r.h.s. of Eq. (3.2) mimics the failure of the DPA upon blowing up the relative correction $\delta_{\mathrm{EW}}^{\mathrm{DPA}}$ by the factor $\left|\sigma_{\mathrm{LO}}-\sigma_{\mathrm{LO}}^{\mathrm{DPA}}\right| / \sigma_{\mathrm{LO}}^{\mathrm{DPA}}$ that is deduced from the LO cross sections based on the full $4 f$ or DPA matrix elements. As we will see below, the last term in $\Delta_{\text {DPA }}$ is surprisingly large in some transversemomentum distributions in the $\mathrm{TeV}$ range.

The quality of the DPA turns out to be excellent, with differences to the full $4 f$ result at the level of some per mille, for integrated cross sections and distributions in angles and rapidities. This fact is illustrated on the 1.h.s. of Fig. 3, where the rapidity distribution of one of the leptons is shown
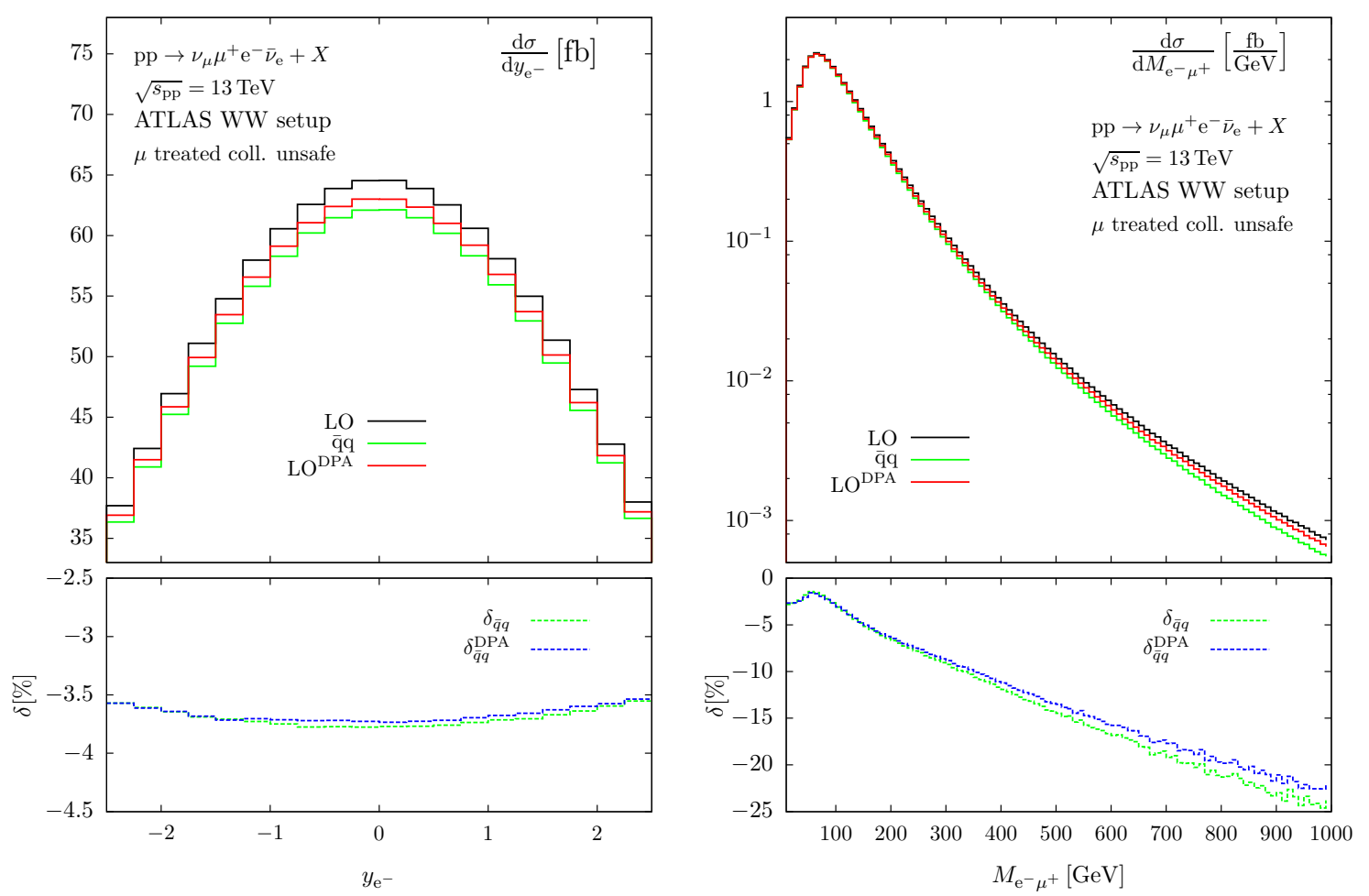

Figure 3: Rapidity distribution of the electron (left) and invariant-mass distribution of the charged-lepton system (right) in pp $\rightarrow v_{\mu} \mu^{+} \mathrm{e}^{-} \bar{v}_{\mathrm{e}}+X$. The lower panels show the relative size of the EW corrections to the $\bar{q} q$ channels compared to the result based on the DPA. (Taken from Ref. [12].) 

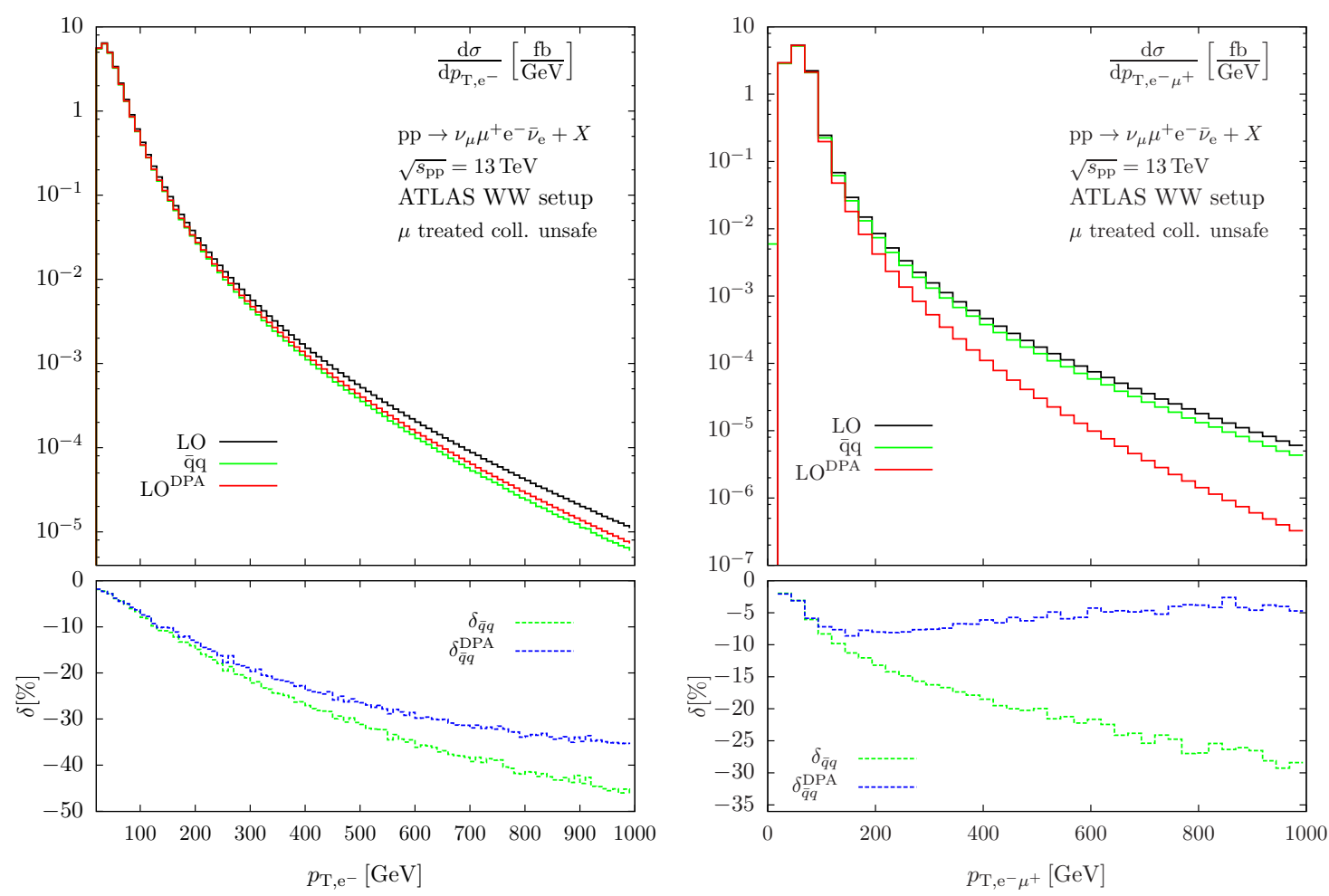

Figure 4: Transverse-momentum distributions of the electron (left) and of the charged-lepton system (right) in $\mathrm{pp} \rightarrow v_{\mu} \mu^{+} \mathrm{e}^{-} \bar{v}_{\mathrm{e}}+X$. The lower panels show the relative size of the EW corrections to the $\bar{q} q$ channels compared to the result based on the DPA. (Taken from Ref. [12].)

together with the corresponding NLO EW corrections (to the $\bar{q} q$ channels) in the ATLAS WW setup. In the upper panel we observe the clear deviation of $\sigma_{\bar{q} q}^{\mathrm{LO}, \mathrm{DPA}}$ from the full LO prediction, being of the same order of magnitude as the EW corrections to the $\bar{q} q$-induced processes. The lower panel shows the excellent agreement of the two versions for the relative corrections, with differences at the $0.1 \%$ level only.

The r.h.s. of Fig. 3 illustrates the same comparison for the invariant-mass distribution of the charged-lepton system. For $M_{\mathrm{e}^{-} \mu^{+}} \lesssim 500 \mathrm{GeV}$, the DPA is accurate within $1 \%$, but the difference grows to about $2-3 \%$ in the $\mathrm{TeV}$ range. This increasing difference between the full $4 f$ calculation and the DPA can already be inferred from the LO cross sections $\sigma_{\bar{q} q}^{\mathrm{LO}}$ and $\sigma_{\bar{q} q}^{\mathrm{LO}, \mathrm{DPA}}$ in the upper panel, which signals the increasing impact of singly-resonant contributions not being included in the DPA. The difference between full and DPA NLO EW corrections is well covered by the last term of our estimate Eq. (3.2). In view of the typically expected accuracies in LHC data analyses, the DPA is certainly sufficient for this observable.

In Fig. 4 we turn to the transverse-momentum distributions of the electron (left) and the charged-lepton system (right). In the $p_{\mathrm{T}, \mathrm{e}^{-}}$distribution, the comparison between full and DPA calculation reveals similar qualitative features as for the $M_{\mathrm{e}^{-} \mu^{+}}$distribution. The differences are, however, larger in size, reaching the 5\% (10\%) level for transverse momenta $p_{\mathrm{T}, \mathrm{e}^{-}}$of about $500 \mathrm{GeV}$ $(1 \mathrm{TeV})$. Again the deterioration of the DPA can already be seen at LO and attributed to an enhanced impact of the singly-resonant background diagrams shown in the second line of Fig. 1, which are 
not included in $\sigma_{\bar{q} q}^{\mathrm{LO}, \mathrm{DPA}}$. The enhancement is due to events where one single lepton is recoiling against the other three in the final state. Thus, for large $p_{\mathrm{T}, \mathrm{e}^{-}}$the cross section $\mathrm{d} \sigma_{\bar{q} q}^{\mathrm{LO}} / \mathrm{d} p_{\mathrm{T}, \mathrm{e}^{-}}$receives large contributions from events where the electron is back-to-back to the three other leptons. For doubly-resonant diagrams (first line in Fig. 1) this situation is less likely for large $p_{\mathrm{T}, \mathrm{e}^{-}}$, where the W-decay lepton pairs mostly appear back-to-back as a result of the boost from the $\mathrm{W}$ rest frames to the laboratory system. The comparison of $\sigma_{\bar{q} q}^{\mathrm{LO}}$ with $\sigma_{\bar{q} q}^{\mathrm{LO}, \mathrm{DPA}}$ at high $p_{\mathrm{T}, \mathrm{e}^{-}}$shows that singlyresonant contributions dominate over doubly-resonant parts already for a $p_{\mathrm{T}, \mathrm{e}^{-}}$of some $100 \mathrm{GeV}$. Kinematically, it is thus easier to produce leptons with high transverse momenta directly rather than through the decay of W bosons. The difference between full and DPA NLO EW corrections is again reproduced quite well by the last term of our estimate Eq. (3.2).

The difference between the full $4 f$ and the DPA calculation is pushed to the extreme in the distribution of the transverse momentum $p_{\mathrm{T}, \mathrm{e}^{-}} \mu^{+}$of the charged-lepton system. Here the enhancement of background diagrams is due to events where one neutrino recoils against the two charged leptons and the other neutrino, a situation that is supported by singly-resonant diagrams, but not by doubly-resonant graphs where the two charged leptons tend to recoil against each other for high transverse momenta.

In conclusion, transverse-momentum distributions are reproduced by the DPA only up to some $100 \mathrm{GeV}$ owing to the growing influence of background diagrams that do not show two simultaneously resonant $\mathrm{W}$ bosons. For predictions of such $p_{\mathrm{T}}$ spectra in the $\mathrm{TeV}$ range, the calculation of EW corrections should be based on a full $4 f$ calculation.

\section{Results on Z-pair production: a Higgs background study}

In Ref. [11] we have discussed EW corrections to $p p \rightarrow \mu^{+} \mu^{-} \mathrm{e}^{+} \mathrm{e}^{-}+X$, focusing on differential cross sections that are particularly interesting for Higgs-boson analyses. In this discussion we divided the EW corrections into photonic and purely weak contributions. Note that this separation respects electromagnetic gauge invariance for neutral-current but not for charged-current processes.

On the 1.h.s. of Fig. 5 we show the invariant-mass distribution of the full four-lepton system, which features the Higgs resonance from gg fusion at $M_{4 \ell} \sim M_{\mathrm{H}} \approx 125 \mathrm{GeV}$ (not included here). The steep shoulder at the Z-pair threshold at $M_{4 \ell}=2 M_{\mathrm{Z}} \approx 182 \mathrm{GeV}$ creates a radiative tail at smaller invariant masses, since $M_{4 \ell}$ can be strongly decreased by final-state radiation effects. A similar effect, though reduced, is observed below the second shoulder near $M_{4 \ell}=110 \mathrm{GeV}$. This is a result of the $p_{\mathrm{T}}$ and invariant-mass cuts $p_{\mathrm{T}}\left(\ell_{i}\right)>6 \mathrm{GeV}, 40 \mathrm{GeV}<M_{\ell_{1}^{+} \ell_{1}^{-}}<120 \mathrm{GeV}$ and $12 \mathrm{GeV}<$ $M_{\ell_{2}^{+} \ell_{2}^{-}}<120 \mathrm{GeV}$, with $\ell_{1}^{+} \ell_{1}^{-}\left(\ell_{2}^{+} \ell_{2}^{-}\right)$refering to the $\ell^{+} \ell^{-}$pair that is closer to (further away from) the nominal mass of the $\mathrm{Z}$ boson. In the region of the Higgs-boson resonance the EW corrections are at the level of a few percent. While photonic corrections might be well approximated by parton showers, this does not apply to the weak corrections. Interestingly, the weak corrections change their size from $-3 \%$ to about $+6 \%$ when $M_{4 \ell}$ drops below the Z-pair threshold. The sign change can be understood from the fact that below the $\mathrm{ZZ}$ threshold one of the two $\mathrm{Z}$ bosons is forced to be far off shell. For the corresponding $\ell^{+} \ell^{-}$pair, its invariant mass $M_{\ell^{+} \ell^{-}}$drops below $M_{Z}$, where the weak corrections are positive, as can be seen in Fig. 1 of Ref. [11]. The sign change of the weak corrections near the $\mathrm{ZZ}$ threshold is quite interesting phenomenologically, since it renders their 

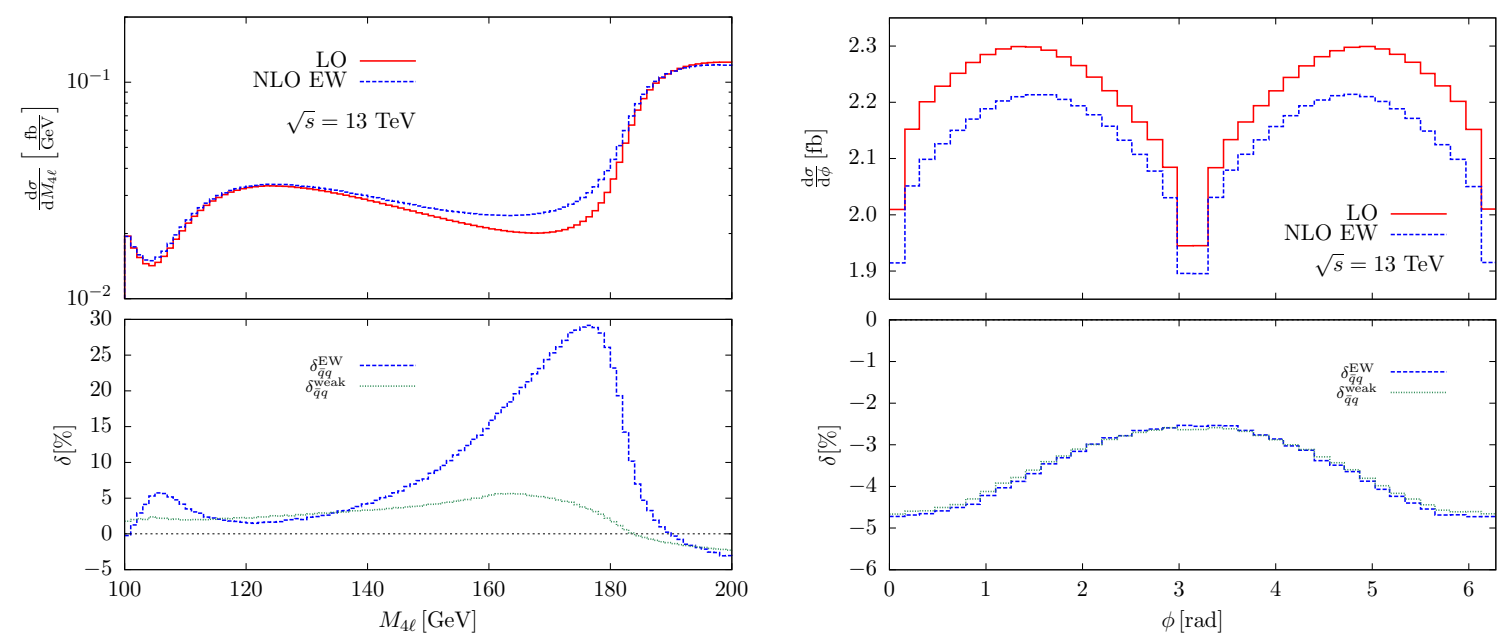

Figure 5: Distributions in the four-lepton invariant-mass and in the angle $\phi$ between the two Z-boson decay planes in $\mathrm{pp} \rightarrow \mu^{+} \mu^{-} \mathrm{e}^{+} \mathrm{e}^{-}+X$ including NLO EW corrections (upper panel), and relative EW and purely weak corrections at NLO (lower panel). (Taken from Ref. [11].)

inclusion via a global rescaling factor impossible. Globally reducing differential cross sections by $3.6 \%$, as deduced from the integrated cross section, would have the opposite effect on the $M_{4 \ell}$ distribution near the Higgs signal as the true weak correction.

On the r.h.s. of Fig. 5 we show the distribution in the angle $\phi$ between the two Z-boson decay planes, which are each spanned by the two lepton momenta of the respective $\ell^{+} \ell^{-}$pair. The distribution is sensitive to possible deviations of the Higgs-boson coupling structure from the Standard Model prediction, so that any distortion of the distribution induced by higher-order corrections, if not properly taken into account, could mimic non-standard effects. The r.h.s. of Fig. 5 reveals a distortion by about $2 \%$ due to weak loop effects. The contribution of photonic corrections is negligible for this observable in our setup. This is due to the fact that photonic corrections mainly influence the absolute size of the lepton momenta via collinear final-state radiation, but not the directions of the leptons.

In summary, the NLO EW corrections to neutral-current four-lepton production consist of photonic and purely weak contributions which display rather different features. Photonic corrections can grow very large, to several tens of percent, in particular in distributions where resonances and kinematic shoulders lead to radiative tails. While those corrections might be well approximated with parton showers, this is not the case for the remaining weak corrections, which are typically of the size of 5\% and, thus, non-negligible. The weak corrections, in particular, distort distributions that are important in Higgs-boson analyses. In the four-lepton invariant mass, even the signs of the weak corrections in the Higgs signal region and the region of resonant Z-boson pairs are different.

Acknowledgements: B.B. and A.D. acknowledge support by the Deutsche Forschungsgemeinschaft (DFG) under reference number DE 623/2-1. The work of M.B., B.J. and L.S. is supported in part by the Institutional Strategy of the University of Tübingen (DFG, ZUK 63) and in part by the German Federal Ministry for Education and Research (BMBF) under contract number 05H2015. S.D. is supported by the DFG through the Research Training Group RTG 2044. The work of L.H. was supported by the grants FPA2013-46570-C2-1-P and 2014-SGR-104, and partially by the 
Spanish MINECO under the project MDM-2014-0369 of ICCUB (Unidad de Excelencia "María de Maeztu"). Part of this work was performed on the high performance computational resources funded by the Ministry of Science, Research and the Arts and the Universities of the State of Baden-Württemberg, Germany, within the framework program bwHPC.

\section{References}

[1] F. Cascioli et al., Phys. Lett. B 735 (2014) 311 [arXiv:1405.2219 [hep-ph]];

T. Gehrmann et al., Phys. Rev. Lett. 113 (2014) no.21, 212001 [arXiv:1408.5243 [hep-ph]];

M. Grazzini, S. Kallweit and D. Rathlev, Phys. Lett. B 750 (2015) 407 [arXiv:1507.06257 [hep-ph]];

M. Grazzini et al., arXiv:1605.02716 [hep-ph].

[2] M. Grazzini, JHEP 0601 (2006) 095 [hep-ph/0510337];

S. Dawson, I. M. Lewis and M. Zeng, Phys. Rev. D 88 (2013), 054028 [arXiv:1307.3249];

Y. Wang et al., Phys. Rev. D 88 (2013) 114017 [arXiv:1307.7520].

[3] P. Nason and G. Ridolfi, JHEP 0608 (2006) 077 [hep-ph/0606275];

S. Höche et al., JHEP 1104 (2011) 024 [arXiv:1008.5399 [hep-ph]];

K. Hamilton, JHEP 1101 (2011) 009 [arXiv:1009.5391 [hep-ph]];

T. Melia et al., JHEP 1111 (2011) 078 [arXiv:1107.5051 [hep-ph]];

R. Frederix et al., JHEP 1202 (2012) 099 [arXiv:1110.4738 [hep-ph]].

[4] F. Cascioli et al., JHEP 1401 (2014) 046 [arXiv:1309.0500 [hep-ph]].

[5] D. A. Dicus, C. Kao and W. W. Repko, Phys. Rev. D 36 (1987) 1570;

E. W. N. Glover and J. J. van der Bij, Phys. Lett. B 219 (1989) 488;

T. Binoth et al., JHEP 0503 (2005) 065 [hep-ph/0503094]; JHEP 0612 (2006) 046 [hep-ph/0611170].

[6] F. Caola et al., Phys. Rev. D 92 (2015) no.9, 094028 [arXiv:1509.06734 [hep-ph]]; Phys. Lett. B 754 (2016) 275 [arXiv:1511.08617 [hep-ph]]; arXiv:1605.04610 [hep-ph].

[7] A. Bierweiler et al., JHEP 1211 (2012) 093 [arXiv:1208.3147 [hep-ph]];

A. Bierweiler, T. Kasprzik and J. H. Kühn, JHEP 1312 (2013) 071 [arXiv:1305.5402 [hep-ph]].

[8] J. Baglio, L. D. Ninh and M. M. Weber, Phys. Rev. D 88 (2013) 113005 [arXiv:1307.4331].

[9] M. Billoni et al., JHEP 1312 (2013) 043 [arXiv:1310.1564 [hep-ph]].

[10] S. Gieseke, T. Kasprzik and J. H. Kühn, Eur. Phys. J. C 74 (2014) 8, 2988 [arXiv:1401.3964 [hep-ph]].

[11] B. Biedermann et al., Phys. Rev. Lett. 116 (2016) no.16, 161803 [arXiv:1601.07787 [hep-ph]].

[12] B. Biedermann et al., JHEP 1606 (2016) 065 [arXiv:1605.03419 [hep-ph]].

[13] A. Denner et al., Nucl. Phys. B 724 (2005) 247 [Erratum-ibid. B 854 (2012) 504] [hep-ph/0505042].

[14] S. Actis et al., JHEP 1304 (2013) 037 [arXiv:1211.6316 [hep-ph]] and arXiv:1605.01090 [hep-ph].

[15] A. Denner, S. Dittmaier and L. Hofer, arXiv:1604.06792 [hep-ph].

[16] A. Denner and S. Dittmaier, Nucl. Phys. B 658 (2003) 175 [hep-ph/0212259]; Nucl. Phys. B 734 (2006) 62 [hep-ph/0509141]; Nucl. Phys. B 844 (2011) 199 [arXiv:1005.2076 [hep-ph]].

[17] S. Dittmaier, Nucl. Phys. B 565 (2000) 69 [hep-ph/9904440];

S. Dittmaier, A. Kabelschacht and T. Kasprzik, Nucl. Phys. B 800 (2008) 146 [arXiv:0802.1405 [hep-ph]].

[18] A. Denner el al., Nucl. Phys. B 587 (2000) 67 [hep-ph/0006307]; Comput. Phys. Commun. 153 (2003) 462 [hep-ph/0209330]. 